\title{
ACUERDOS Y COMPROMISOS: FAMILIAS Y PROGRAMAS SOCIALES DESDE UNA MIRADA TEÓRICA ${ }^{1}$
}

\author{
AGREEMENTS AND COMMITMENTS: FAMILIES AND SOCIAL \\ PROGRAMS FROM A THEORETICAL PERSPECTIVE
}

\author{
Florencia Picasso Risso ${ }^{2}$ \\ fpicassorisso@gmail.com
}

Fecha de recepción: 13 junio 2016 - Fecha de aceptación: 9 enero 2017

\begin{abstract}
Resumen
El presente artículo procura contribuir a la reflexión teórica sobre los encuentros entre las familias beneficiarias y los programas sociales en el contexto latinoamericano. Se busca reflexionar cómo se produce la recepción de las políticas sociales, en relación a los sujetos vulnerados en sus derechos y qué sucede a partir de tales intervenciones. Desde esta premisa inicial vinculada a la tesis doctoral, parte el presente artículo que procura desarrollar una aproximación teórica a estas interrogantes, a la luz de la mirada de Habermas, Bourdieu y Berger y Luckmann. La reflexión despierta varias pistas teóricas atrayentes para tensionar las prácticas, expectativas, posibilidades e imposiciones, desde la lógica familiar-institucional-profesional y en el marco de la intervención de la política pública. En este sentido, resulta una tríada potente para abordar el objeto de estudio.

Palabras clave: Políticas sociales, Tensiones, Intervención Profesional, Poder, Interacción.
\end{abstract}

\begin{abstract}
This article contributes to the analysis of the theoretical reflection on the "beneficiary families' gatherings and social programs in the Latin American. Moreover, it researches on the reception of social policies related to the subjects, whom their rights are violated, and the findings of such interventions. From this initial premise, related to the doctoral thesis, part of this essay seeks to develop a theoretical approach to these questions, under the perspective of Habermas, Bourdieu and Berger, and Luckmann. The reflection awakens several attractive theoretical clues to stress the practices, expectations, possibilities and impositions, from the familiar-institutional-professional logic and within the framework of public policy intervention. In this sense, it is a powerful triad to tackle the object of study.
\end{abstract}

Keywords: Social policies, Tensions, Professional intervention, Power, Interaction.

1 La naturaleza del artículo se trata de una reflexión teórica a partir de las interrogantes planteadas en mi anteproyecto de tesis doctoral en el marco del Doctorado en Sociología de la Universidad Alberto Hurtado, Chile. No se trata de resultados de investigación sino de aproximaciones teóricas, a la luz de Habermas, Bourdieu y Berger y Luckmann, por considerarse una tríada potente para la comprensión a los encuentros entre las familias y los programas sociales. Resulta atrayente para tensionar las prácticas, expectativas, posibilidades e imposiciones, desde la lógica familiar-institucional-profesional y en el marco de la intervención de la política pública. Trabajo realizado gracias a una beca de la Comisión Nacional de Investigación Científica y Tecnológica (CONICYT), Doctorado Nacional, Chile, 2016. 


\section{Introducción}

En los últimos años se han producido transformaciones en las políticas sociales en el contexto latinoamericano, que cuestionan el lugar que ocupan los sujetos tanto en las definiciones operativas, el diseño, la gestión, la ejecución, la participación y las formas de monitoreo y evaluación (Gradin, Picasso y Rieiro, 2012). Estos cambios han generado un nuevo abordaje al momento de reflexionar sobre las políticas sociales, donde el sujeto comienza a incorporar múltiples dimensiones: sociales, culturales, políticas, dejando de lado la económica como preponderante. Este abordaje integral sobre el sujeto, junto con la consideración de su propio contexto, permite transformarlo en un sujeto activo capaz de modificar su propio destino (Serna, 2012). Las familias en este contexto emergen como objeto de estudio, unidades de análisis, y de intervención orientando políticas cuyo foco es la vulnerabilidad social en la cual se encuentran inmersas (Arriagada, 2001, Picasso, coord.2010).

En este artículo se realiza una reflexión teórica a partir de algunas interrogantes sobre dos programas dirigidos a las familias del contexto latinoamericano: el Programa Familias, del Sub Componente Seguridades y Oportunidades en Chile y la Estrategia Nacional de Fortalecimiento de las Capacidades Familiares, Programa Cercanías en Uruguay, en cuanto a la nueva modalidad de programas denominados de cercanías ${ }^{3}$ que procuran la interinsitucionalidad, el abordaje territorial, y un dispositivo de proximidad de los equipos técnicos territoriales o apoyos familiares, hacia el abordaje de las familias. Estos aspectos constituyen desafíos que procuran el diálogo en términos colaborativos entre la ciudadanía y las instituciones de bienestar. Este abordaje de la política social constituye una novedosa herramienta para los programas sociales que trabajan con situaciones de vulnerabilidad desde la perspectiva del uso y apropiación, por parte de las familias beneficiarias de los mismos.

Resulta relevante plantear algunas características de ambos contextos siguiendo a Antía (2008), luego de la fase de reformas liberales en la región, el acceso al gobierno de partidos de izquierda y centro izquierda despertó expectativas en relación a las transformaciones que estos promoverían. El Estado social chileno conserva una configuración predominantemente liberal, aunque con un cierto fortalecimiento de los dispositivos solidarios; al tiempo que el uruguayo presenta una estructura "híbrida", manteniendo una mayor presencia relativa de mecanismos colectivos en el sistema de protección social.

Concretamente, se produce un intento por generar programas focalizados que atacan las grietas de los regímenes contributivos, para poder alcanzar cobertura universal y segmentada (Filgueira, 2013). Una forma de analizar la temática es la caracterización de regímenes híbridos (Antía, 2008) que se ha propuesto para el caso uruguayo. Ello es particularmente cierto para los países con regímenes de bienestar maduros o más desarrollados. La economía política del giro a la izquierda ${ }^{4}$ ayuda a entender, parcialmente, estas dinámicas.

\section{Principales preguntas y aproximación teórica a las interrogantes desde la mirada de los autores}

En este marco, tomando como punto de partida la interacción entre las familias y los equipos técnicos de los programas. Algunas de las interrogantes que se plantean son: ¿Cómo se da el vínculo

3 Refieren a la conformación de dispositivos instrumentados por profesionales del área psico- social, cuyo objetivo es el acompañamiento de los núcleos familiares para gestionar las situaciones asociadas a la extrema pobreza y la indigencia mediante las visitas domiciliarias y la vinculación y derivación a otras políticas institucionales existentes en el plano de la identificación civil, los cuidados, salud, la educación, la atención pública a la infancia, la capacitación laboral, el empleo asistido y los microcréditos sociales (Mides, 2012, Fosis, 2006).

4 Se trata en América Latina de los contenidos de las agendas post-Consenso de Washington y la recuperación de las capacidades estatales. La idea de postneoliberalismo y el denominado "retorno del Estado" resultan elementos indispensables de diferentes interpretaciones conceptuales y políticas. 
entre la política social y las familias beneficiarias? ¿Cómo se abordan los acuerdos/compromisos entre la política social y las familias? ¿Cómo se establecen las relaciones de poder internamente en las mismas con relación a los acuerdos o compromisos alcanzados?

Cabe señalar, que un hallazgo relevante desde el abordaje comparativo de los programas dirigidos a las familias, es la aproximación al proceso de "acompañamiento familiar" que desarrollan los equipos territoriales -en Uruguay- y los apoyos familiares -en Chile-, en función de cada Plan Familiar -elaborado entre el equipo territorial/apoyo familiar y cada familia, contemplando aquellos derechos vulnerados-. De esta forma, siguiendo a Angulo y Marques (2015) los equipos técnicos de los programas, como dispositivo de proximidad, buscan problematizar la vida cotidiana de las propias familias, desnaturalizando situaciones invisibles de poder y buscando estrategias que procuren resignificar y promover mejoras en las condiciones de vida.

Dando cuenta del abordaje se desarrollan algunas categorías conceptuales vinculadas al corpus de la concepción teórica de los autores que permiten tensionar los textos desde las preguntas formuladas. En primer lugar, desde la teoría de la acción comunicativa de Habermas (1999) y su abordaje contemporáneo del mundo de la vida y sistema. Asimismo, desde el concepto de dominación y abordaje del poder en Bourdieu y la mirada fenomenológica de Berger y Luckmann aludiendo a los procesos de institucionalización y legitimación. De esta forma, se realiza la aproximación a los encuentros entre las familias beneficiarias, y la política social abordando las características que poseen el poder y las diferentes lógicas y racionalidades imperantes que subyacen en los mismos.

Resulta iluminadora la teoría de la acción comunicativa habermasiana (1999). La misma aporta la mirada histórica y dialéctica macro-sociológica que, permite contribuir al desarrollar un marco atractivo para comprender la interacción social a nivel micro tal como se propone este artículo.

El autor formula una aproximación teórica de análisis de la sociedad que pone de manifiesto dos racionalidades: la racionalidad sustantiva del mundo de la vida y la racionalidad del sistema. El mundo de la vida representa una perspectiva interna, la de los actores que se desenvuelven en la sociedad, mientras que el sistema representa la perspectiva externa, estructura sistémica donde coexiste la racionalidad técnica, burocratizada -aludiendo al enfoque weberiano- de las instituciones.

El concepto abstracto de mundo es condición necesaria para que los sujetos que actúan comunicativamente puedan entenderse entre sí sobre lo que sucede en el mundo o lo que hay que producir en el mundo. Con esta práctica comunicativa se aseguran a la vez del contexto común de sus vidas, del mundo de la vida que intersubjetivamente comparten (Habermas, 1999).

En concreto, en la categoría conceptual del "mundo de vida" se visualizan relaciones de entendimiento y asociatividad. Se basa en un proceso cooperativo de interpretación en que los participantes se refieren, simultán do. Esto implica, por un lado, la 'autonomización' creciente del sistema político cuyas comunicaciones se enlazan mediante el uso del poder como medio de intercambio, coordinación y control. Este intercambio resulta similar al que se realiza a través del dinero en los intercambios económicos. Desde esta perspectiva el poder permitiría la coordinación en un ámbito funcionalmente diferenciado: el ámbito político; y resultaría específico y delimitado.

En el marco de su teoría Jürgen Habermas (1999) reconoce y desarrolla la tesis weberiana partiendo de que toda acción del individuo está orientada en función de los fines que rigen a partir de acciones instrumentales y estratégicas. Ante este abordaje plantea el concepto de acción comunicativa, el cual describe a individuos que hacen uso de la racionalidad comunicativa, no con un objetivo meramente orientado a fines propios, sino, más bien, con el objetivo del entendimiento. De esta forma, el autor procede a identificar, por un lado, una acción, en un sentido estricto, - acción no lingüística- que tiende a la consecución de un fin preconcebido por el actor en el mundo; por otro lado, las expresiones lingüísticas descritas como acciones que se orientan a la comprensión y entendimiento mutuo de algo acerca del mundo: "El científico social cae bajo la perspectiva del agente mismo. Tenemos que aclarar en qué sentido queda con ello introducido el entendimiento lingüístico como un mecanismo de coordinación de la acción" (Habermas, 1999, p.36). 
Siguiendo al autor la acción comunicativa, posee como componente clave, la coordinación a través del uso del lenguaje. En este marco, se encuentran de forma implícita la competencia comunicativa, forma ideal de habla, consenso, validez del habla, entre otros. Con estos supuestos identifica los conceptos necesarios para analizar las estructuras generales del actuar orientado hacia la comprensión.

Desde su corpus teórico es posible ensayar algunas aproximaciones y respuestas a las preguntas formuladas. Es así que, desde el abordaje del mundo de la vida y el sistema social se brinda una macro- explicación, que alude a cómo la acción estratégica con arreglo a fines se impone sobre la acción comunicativa que busca el acuerdo y el consenso. Desde la explicación histórica del desacoplamiento, entre mundo de la vida y sistema social y la colonización del mundo de la vida. En este sentido, permite reflexionar y formular una aproximación a las distintas lógicas imperantes en la interacción concreta de los encuentros señalados. Por un lado, la lógica de ambos programas dirigidos a las familias en Uruguay y Chile que procuran entretejer ciertos "acuerdos y compromisos" con éstas, fomentando una interacción similar a la de una esfera pública ampliada; como un espacio que promueva o habilite que las familias participen en la construcción de sus proyectos de vida. Por otro, las tensiones resultantes de la respuesta de las familias a la intervención de la política, que conlleva a nuevas interrogantes en relación a si efectivamente se gesta un acción comunicativa real a través de los "acuerdos y compromisos" formulados en donde:

Hablantes y oyentes se refieren desde el horizonte preinterpretado que su mundo de la vida representa, simultáneamente a algo en el mundo objetivo, en el mundo social y en el mundo subjetivo para negociar definiciones de la situación que puedan ser comprensibles por todos (Habermas, 1992, p.134).

Asimismo, cabe preguntase también, si en dicha esfera de interacción se da una lógica de poder que invisibiliza las necesidades de las familias y procura impulsarlas artificialmente a responder tanto al Estado o al Mercado y sus diversas lógicas en función de los regímenes de bienestar.

Es así, que resultaría imperioso indagar si en dicho escenario, donde la política comienza a ser co-gestionada: ¿existió un cambio hacia un accionar estratégico, no consumándose efectivamente la acción comunicativa de entendimiento mutuo? o por el contrario, ¿se trata de una ampliación del mundo de la vida o de la esfera pública; o en realidad permea una lógica de poder que coloniza uno sobre otro?

Estas interrogantes planteadas permiten comprender el encuentro entre las familias y los equipos técnicos/apoyos familiares donde se realizan los compromisos -en el contexto Chile mercadocéntrico- y acuerdos en el contexto uruguayo -estadocéntrico e híbrido-. Si bien, no se realiza en ambos programas transferencias monetarias condicionadas, si predominan las contrapartidas vinculadas a las necesidades e intereses que se acuerdan entre las familias y los equipos técnicos, a través de los planes familiares. Para recibir, de esta forma, el apoyo psicosocial; así como: servicios de cuidado, salud, nutrición, educativos, laborales, sociales culturales, y hasta en ocasiones la participación en fondos concursables para emprendimientos. Así, se puede visualizar también como se tensionan las lógicas en ocasiones contrapuestas entre los equipos técnicos/apoyos familiares y la política gubernamental con sus objetivos previamente establecidos.

Existen múltiples racionalidades distintas que se plasman en tensiones resultantes entre el ejercicio del poder político, los equipos técnicos y el respeto por la autonomía de las familias. Tales reflexiones sobre estos límites del poder se pueden visualizar: en las lógicas profesionales y racionalidades distintas que guían la forma de abordaje hacia las familias.

Para realizar una segunda aproximación a las formas implícitas del poder en juego en los encuentros entre las familias y las instituciones de bienestar, ahora desde la mirada teórica de Bourdieu, se hace necesario plantear su conceptualización sobre el poder, considerándolo como un tipo de relación entre individuos constitutiva en la sociedad. En este sentido, el autor $(1991,2012)$ plantea el carácter relacional de la sociedad. Las sociedades están conformadas por relaciones de fuerza en distintos espacios sociales. El mundo social está organizado como un sistema de relaciones de fuerza donde los agentes actúan 
según la posición en el interior de dicho espacio y asimismo, sobre los frutos del campo que sobre ellos ejercen las mismas luchas y relaciones de fuerza que constituyen el propio campo.

Desde esta mirada tanto la familia como las instituciones de bienestar con sus equipos técnicos/ apoyos familiares, posee sus propias relaciones de poder a la interna condicionada por los códigos existentes en cada campo, incluyendo los profesionales aplicados por los equipos técnicos. De esta manera, se visualiza como el poder, el conocimiento, la intervención y la lógica profesional están estrechamente ligados en dicho encuentro entre las familias y el Estado. La intervención está, fundamentalmente, mediatizada por los códigos profesionales aplicados por los equipos técnicos que a su vez se encuentran tensionados con la lógica política.

La construcción teórica de Bourdieu, parte la reflexión y análisis en las estructuras sociales de dominación simbólica a través de la violencia simbólica, lo que resulta clave para abordar las interrogantes. De este modo, realiza una síntesis de las perspectivas estructuralistas desde el actor.

La Distinción (2012) aborda los usos sociales de la cultura como instrumento de dominación simbólica, estableciendo así, quienes son incluidos y excluidos en la sociedad; esto en función de sus bienes materiales y simbólicos, reglas, significados y necesidades y valores de su grupo de pertenencia. En este sentido, se visualizan algunas reflexiones vinculadas al campo de disputa simbólica donde se representa la división de clases y las acciones colectivas para combatirla o potenciarla, las desigualdades de capital cultural y el sacrificio personal vinculado a las competencias estéticas.

Siguiendo a Bourdieu (2012) el pensamiento de los actores se trata de un componente más de las relaciones objetivas. Al introducir lo que los actores perciben y sus definiciones, se constituye una visión de lo social, más articulada y compleja que permite la comprensión del funcionamiento de la sociedad: "el habitus es a la vez, en efecto, el principio generador de prácticas objetivamente enclasables y el sistema de enclasamiento (principium divisionis) de esas prácticas" (Bourdieu, 2012, p.200).

La distinción necesaria para el ejercicio dominante del poder segrega a las formas peculiares de vida e incluye a los dignos. Desde esta conceptualización se expresan las reglas distintivas del poder como referencia donde se construye la visión del "alter". En el caso de las familillas en situación de vulnerabilidad, resulta interesante reflexionar siguiendo al autor, en tanto "grupos marginales o excluidos" (Bourdieu, 2012, p.184) así como del habitus concreto de la marginalidad.

Asimismo, reflexionar sobre si las prácticas existentes, se reproducen y cómo el encuentro entre las familias y los equipos técnicos busca generar nuevas prácticas que puedan construirse a través de las significaciones de las familias. También, distinguir si los programas y equipos, realmente, han podido modificar sus hábitos conductas, etcétera; y plantear interrogantes y tensiones mayores en relación al sostén para la inclusión social, pretensión de las políticas sociales a el largo plazo. ¿Existe un cambio de las propias prácticas que auto-reproducen la exclusión, hay algún sistema de sostén detrás o las estructuras en efecto reproducen su propia exclusión?

Resulta interesante, abordar empíricamente las características que puede tener una posible contra-intervención, planteada como abordaje en cuanto a las resistencias que se generan en la intervención de los equipos técnicos/ apoyos familiares. No se trata de los obstáculos planteados únicamente desde las familias, sino desde las propias instituciones de bienestar burocráticas. Allí, se produce otra tensión entre las prácticas cotidianas, las estructuras- estructurantes en referencia a la subjetivación y ¿cómo estos espacios de intercambio entre los técnicos con las familias puede modificar o generar nuevas prácticas, nuevas construcciones sociales de la realidad - tal como se señalará en términos de Berger y Luckmann- y asimismo, considerar: ¿cómo el sistema acoge esos cambios o los reproduce en las estructuras de poder desiguales que caracterizan a las propias sociedades diferenciadas?

En este sentido, aludiendo al cuestionamiento, la dominación simbólica tal como subraya Goineix (2012) aludiendo a Bourdieu (2012), es ejercida en el ámbito personal y afectivo, debido a que la autoridad es percibida como característica de la persona que la lleva adelante. Solo con su institucionalización es posible disminuir costos de su reproducción constante, la manera de reproducir capital simbólico de forma duradera y a bajo costo. Según el autor uno de los mecanismos citado como ejemplo es "el 
mercado de las titulaciones", que asigna esa legitimidad a la titulación académica, pues con el sistema educativo la dominación se estabiliza, se perpetúa y concede legitimidad.

En tercer lugar, la aproximación a las interrogantes planteadas desde Berger y Luckmann (2001), se realiza desde una mirada fenomenológica, de la construcción social de la realidad, desde una aproximación a la vida cotidiana del sujeto. Se trata de una teoría de la sociología del conocimiento que procura realizar una síntesis de varios autores clásicos de la sociología. La tesis central de los autores señala: "Lo que afirmamos es fundamentalmente, que la realidad se construye socialmente y que la tarea de la sociología del conocimiento es en analizar los procesos por los que ocurre tal cosa" (Berger y Luckmann, 2001, p. 13).

Se inspiran en Schütz, en relación con las realidades múltiples y consideran a los conocimientos de la vida cotidiana como los más reales, los cuales se imponen con más fuerza, debido a que se da por supuesto sin cuestionamiento a priori. Este fenómeno se manifiesta a través de las interacciones, la negociación, leguaje, las estructuras de pertenencia a través de códigos y signos compartidos por un conjunto de sujetos en un espacio-tiempo común, singular y por ellos reproducido.

La sociedad se construye a partir de la secuencia de exteriorización-objetivación, a través de las realidades producidas por las acciones humanas como externas a ella. Para ello, resulta relevante comprender dos procesos: la institucionalización y la legitimación.

El concepto de "institucionalización" alude a cuando los hábitos necesarios para el accionar humano adquieren permanencia independiente de los individuos concretos: "La institucionalización aparece cada vez que se da una tipificación recíproca de acciones habitualizadas por tipos de actores. Dicho en otra forma, toda tipificación de esa clase es una institución” (Berger y Luckmann, 2001, p.76).

La legitimación se da a través de "los universos simbólicos" como: "cuerpos de tradición teórica que integran zonas de significado diferentes y abarcan el orden institucional en una totalidad simbólica" (Berger y Luckmann, 2001, p.124). Los autores explican de esta forma al orden institucional como: estable y conocido.

La construcción social de la realidad se realiza a través de la interiorización a través de los procesos de socialización primaria y secundaria, consiguiendo simetría en la realidad subjetiva del individuo y la objetiva definida por la sociedad. Sin lugar a duda las familias son importantes en este proceso, pero, asimismo, se advierte desde el punto de vista de los autores, la posibilidad que la socialización fracase vinculada a situaciones estructurales como la división social del trabajo.

Esta aproximación a los autores permite en particular abordar el concepto de "universos simbólicos", que dan forma al encuentro entre las familias y los profesionales en la orientación a los planes familiares traducidos en los "acuerdos o compromisos".

Retomando la pregunta planteada anteriormente de: ¿cómo estos espacios de intercambio entre los técnicos con las familias puede modificar o generar nuevas prácticas, nuevas construcciones sociales de la realidad?

Asimismo, desde la postura de los autores se rescata la posibilidad de construcción de proyectos de vida por parte de las familias, a través de procesos de "resocialización o alternación" que pueden cambiar radicalmente su realidad subjetiva y objetiva (Berger y Luckmann, 2001, p.197) a través de una base social que sirva de laboratorio.

\section{Tensiones y contrastes a la aproximación de los autores}

Desde la lectura crítica de Bourdieu (2012) se plantea el carácter transversal de las relaciones de poder, por tanto no existiría un ámbito exento de relaciones de poder en el cual fluya lo social de forma genuina, siempre se encuentran en relación al campo de relaciones de fuerza. Aspecto que, contrasta con Habermas en su conceptualización de "acción comunicativa". 
Ambos poseen diferentes formas de concebir el poder: Bourdieu lo visualiza como relaciones de fuerza y Habermas como mecanismo de coordinación que opera en un sistema funcionalmente diferenciado.

Tal como subraya Aguilar (2014) siguiendo a Habermas (1999) el poder se trata de un mecanismo que sustituye al lenguaje como manera de coordinar acciones prescindiendo del entendimiento comunicativo. Es un mecanismo sistémico que hace posible estabilizar redes de acción sin que dependan del acuerdo entre los sujetos. Asimismo, no contempla el poder, al igual que sucede con el dinero, "sustituye al entendimiento lingüístico como mecanismo de coordinación” (Habermas, 1999, p. 473) sin tener el anclaje institucional en el mundo de la vida, el cual se expresa en el marco institucional que regula la operación del medio de control. Al igual que sucede con el vínculo entre la acción comunicativa y la estratégica, pues el modo original de uso del lenguaje es el comunicativo, y el resto de los usos no comunicativos, resultan secundarios al no tender al lenguaje.

Siguiendo el pensamiento de Habermas (1992) las relaciones de poder son diferentes que las relaciones sociales que se coordinan por el medio del entendimiento comunicativo y asimismo, terminan resultando nocivas ya que sustituyen el entendimiento por el poder. Esto corresponde a lo que el autor conceptualiza como colonización sistémica del mundo de la vida, donde la expansión de los sistemas claramente diferenciados perturba los procesos de reproducción simbólica del mundo de la vida. Esta perturbación e interferencia se realiza por el uso del dinero y el poder en los ámbitos de acción estructurados comunicativamente. Desde su enfoque, al considerar la base de la sociedad en los ámbitos estructurados normativamente y comunicativamente, sitúa a las relaciones de poder como exteriores a las relaciones comunicativas.

Una crítica al concepto habermasiano de la acción comunicativa, es que no se focaliza en las desigualdades, ni tampoco en disputas de poder. Este se trata de un punto de convergencia con la aproximación de Berger y Luckmann, ya que estos últimos autores, no realizan un aporte contundente en este sentido, aspecto que la distancia de Bourdieu. En el caso de Berger y Luckmann sistematizan los enfoques de Weber sobre el poder y se articula con la perspectiva de Schütz. Se utiliza la noción de poder desde varias aristas diferentes: en los procesos de aprendizaje, desde la existencia de la superioridad de las instituciones y desde la conceptualización de poder político desde la dominación weberiana: "la probabilidad de imponer la propia voluntad, dentro de una relación social, aún contra toda resistencia y cualquier que sea el fundamento de esa probabilidad" (Weber, 1987, p.43). En la aproximación en la vida cotidiana, se pueden plantear algunos vacíos o interrogantes en relación al papel de la socialización en cuanto a que la realidad objetiva adquiere cierto carácter de coacción.

Asimismo, poseen puntos coincidentes en cuanto a los elementos de reproducción simbólica del mundo de la vida según Habermas y de la vida cotidiana en la construcción social de la realidad en Berger y Luckmann. Siguiendo a los autores, la vida cotidiana se basa en la interacción cuya forma más importante es el idioma y el saber que se desprende de este. Se trata de la posibilidad de que todos puedan comprender el mundo cotidiano-objetivación. En el caso de Habermas sería a través del lenguaje como forma de llegar al entendimiento en el mundo de la vida. Pero también, poseen puntos tensionantes en relación al concepto de reificación de los fenómenos humanos como si fueran cosas (Berger y Luckmann, 2001) y en relación al concepto de dialéctica en tanto configuración socio-histórica en el caso de Habermas e interacción recíproca según Berger y Luckmann.

Articulando la perspectiva de los autores, Bourdieu (1997), concibe al campo familiar y la familia como cuerpo. En la segunda acepción se vincula al concepto fenomenológico como principio de construcción socialmente construido. Asimismo, a la interna de las familias, la distribución de los recursos, poder, invisibiliza a algunos miembros de menor poder como los niños, las niñas y adolescentes en el marco de las relaciones familiares y de los propios acuerdos/compromisos generados con los programas.

Finalizando, este apartado, y retomando a Bourdieu, las estructuras de dominación corresponden a la objetivación de las disposiciones-hábitus- incorporados en los agentes y por tanto no resultan modificables mediante la acción reflexiva de los sujetos aspecto que lo tensiona con Habermas. Es así que, 
en su conceptualización del habitus, en tanto "estructura- estructurada" (2012, p.201), se plantean nuevas tensiones, desafíos y preguntas: ¿sería viable quebrantar estas estructuras de dominación en clave de promover reales capacidades de agencia por parte de las familias? ¿Cómo sería posible concretar propuestas contra-hegemónicas que redistribuyan el poder y los recursos hacia quienes son más vulnerables? ¿Sería ingenuo pensar en una esfera pública que promueva propuestas que valoren la diversidad, de conocimientos, y experiencias y así convivir hacia modelos democráticos dialógicos donde se respeten las visiones y significaciones, sin colonizar una sobre otras?

Estas nuevas interrogantes son fundamentales para comprender en profundidad, la real capacidad de agencia de las familias, las respuestas desde sus propias significaciones, la forma de reinterpretar y dar respuesta a la política social, así como, las expectativas de transformación que ésta tiene sobre las mismas.

\section{Algunas Consideraciones finales}

En este último apartado se plantean algunas reflexiones finales sobre el abordaje planteado, indicando algunas limitantes, potencialidades y desafíos.

La aproximación teórica trazada para abordar las interrogantes planteadas permitió evidenciar las tensiones contradicciones y formular nuevas preguntas relevantes para el abordaje. El poder ejercido en los encuentros entre las familias y las instituciones de bienestar en el contexto latinoamericano y en particular en ambos programas dirigidos a las familias seleccionados en Chile y Uruguay, no se da de forma uniforme, coherente o predeterminada.

Tal como se planteó en el análisis, los programas se encuentran permeados por una serie de lógicas y prácticas profesionales y políticas distintas; en ocasiones contradictorias. Se hace necesario tener en cuenta sus diferentes racionalidades, lógicas y significaciones.

La intervención está, primordialmente, condicionada por los códigos profesionales aplicados por los equipos técnicos/apoyos familiares que a su vez se encuentran tensionados con la lógica política. En particular, los marcos discursivos y prácticos dan forma al encuentro entre las familias y los profesionales en la orientación a los planes familiares que se concretan en acuerdos o compromisos. De esta manera, el poder, el conocimiento, la intervención y la lógica profesional están estrechamente ligadas en dicho encuentro entre las familias y los programas (Mik-meyer y Villadsen, 2014). Desde mi punto de vista, se trata de un desafío; el construir nuevas prácticas que logren deconstruir, los proyectos de vida de las familias y toma de decisiones para ser realmente sujetos activos, capaces de modificar su propio destino tal como se mencionaba al inicio del presente artículo.

Coincido con la perspectiva de Bourdieu, el poder en este contexto de interacción debe ser visto como transversal a las relaciones e inmanente a su contexto y no debe ser considerado como primordial, unidireccional o esencialmente represivo.

Resulta interesante reflexionar siguiendo a Bourdieu, las familias en situación de vulnerabilidad, en tanto "grupos marginales o excluidos" (Bourdieu, 2012, p. 184); así como, del habitus concreto de la marginalidad. Indagando acerca de las prácticas existentes, si se reproducen y cómo el encuentro entre las familias y los equipos técnicos busca generar nuevas prácticas que puedan construirse a través de las significaciones de las familias.

Siguiendo a Berger y Luckmann (2001) la legitimación se da a través de los "universos simbólicos", que dan forma al encuentro entre las familias y los profesionales en la orientación a los planes familiares traducidos en los "acuerdos o compromisos". Desde esta postura, se rescata la posibilidad de construcción de proyectos de vida por parte de las familias, a través de procesos de "resocialización o alternación" que pueden cambiar radicalmente su realidad subjetiva y objetiva a través de los programas, lo cual no implica que se encuentre exenta de tensiones y contradicciones.

Como desafíos de investigación resulta relevante considerar la complejidad de aristas que encierra la problemática: el contexto emergente del nuevo escenario de las políticas sociales y las nuevas modalidades vinculadas a la interinstitucionalidad, la participación, la territorialidad y los 
dispositivos de proximidad de los equipos técnicos/apoyos familiares, las familias y las tensiones resultantes entre ellas.

En este sentido, tiendo a identificarme con la perspectiva de Bourdieu, en tanto concebir el poder como relaciones de fuerza; asimismo aproximaciones de abordaje constructivistas y predominantemente post-estructurales desde la idea de que el poder debe ser visto como algo intrínseco a las relaciones sociales. Pero me resulta desafiante pensar en propuestas contra-hegemónicas que partan de modelos democráticos dialógicos que posibiliten el diálogo la diversidad, de conocimientos, y experiencias procurando la redistribución del poder y los recursos hacia los colectivos más vulnerables.

En síntesis, desde el abordaje de este ensayo como primera aproximación teórica a la temática de tesis doctoral, despierta varias pistas teóricas que pueden iluminar el análisis. La articulación de lo abordajes entre Habermas, Bourdieu y Berger y Luckmann resulta atrayente para tensionar las prácticas, expectativas, posibilidades e imposiciones, desde la lógica familiar-institucional-profesional y en el marco de la intervención de la política pública. En este sentido, resulta una tríada potente para abordar el objeto de estudio.

En relación a las diferencias de poder a la interna de las familias, se requiere integrar la perspectiva de género, generaciones y cuidados que permita develar dichas relaciones de poder implícitas y las no implícitas y su vínculo con los respectivos "acuerdos y compromisos". Resulta interesante reflexionar en esta clave teórica, pensando qué aporta "generación", qué no aporta "parentesco" desde la formulación de nuevas preguntas que iluminen el abordaje.

Estas consideraciones finales resultan fundamentales para la búsqueda del diálogo entre el abordaje teórico, desarrollado en este ensayo, y el metodológico, aspectos imprescindibles desde el oficio del sociológico. Para ello, es importante desarrollar una actitud de "ruptura y vigilancia epistemológica" en términos de Bourdieu et. al (1975), que permita proponer "el principio de la no conciencia" que implica ser conscientes de lo que parecería obvio y contar con la disposición de problematizar y reformular los conceptos con base en las nuevas dimensiones o repercusiones que se pueden describir en el objeto de estudio.

\section{Bibliografía}

Aguilar, Omar. (2014). El poder de la sociedad: Una lectura sociológica de Michel Foucault. En Figueroa, Maximiliano (Ed). Poder y Ciudadanía Estudios sobre Hobbes, Foucault, Habermas y Arendt. Santiago: Ril.

Cercanías, Estrategia Nacional de Fortalecimiento de las Capacidades Familiares. (2015). Modelo de Atención, Montevideo: Ministerio de Desarrollo Social (MIDES).

Angulo, Sofía y Marques, Agustina. (2015). Estrategia de Intervención en Cercanía. Las nuevas políticas sociales de articulación en territorio. El caso de Cercanías en la región norte de Uruguay. En Serna, (Coord.), Cuadernos de Ciencias Sociales y Políticas Sociales 1, (pp.197-216). Montevideo: MIDES, Universidad de la República (UdelaR).

Antia, Florencia. (2008). Transformaciones del Estado Social: ¿Hacia una nueva fase de reformas en Chile y Uruguay? Revista Debates, v.2, (n.1): 123-149.

Arriagada, Irma. (2001). Familias latinoamericanas. Diagnóstico y políticas públicas en los inicios del nuevo siglo. Santiago de Chile: Comisión Económica para América Latina y el Caribe (CEPAL).

Berger, Peter y Luckmann, Thomas. (2001). La construcción social de la realidad. Buenos Aires: Amorrortu.

Bourdieu, Pierre; Passeron, Jean-Claude y Chamboredon, Jean-Claude. (1975). El oficio del sociólogo. Buenos Aires: Siglo XXI.

Bourdieu, Pierre. (1991). El sentido práctico. Madrid: Taurus.

Bourdieu, Pierre. (1997). El espíritu de familia. En Razones prácticas. Sobre la teoría de la acción. Barcelona: Anagrama. 
Bourdieu, Pierre (2012). La distinción. Criterios y bases sociales del gusto. Madrid: Taurus.

Filgueira, Fernando (2013). Los regímenes de bienestar en el ocaso de la modernización conservadora: posibilidades y límites de la ciudadanía social en América Latina. Revista Uruguaya de Ciencia Política, 22 (2): 17-46.

Fondo de Solidaridad e Inversión Social, (FOSIS). (2006). Estudio sobre un Modelo de Desarrollo de Autonomía y ejercicio de la ciudadanía de las personas y familias participantes del Programa Puente a través del análisis y evaluación de sus prácticas, interpretaciones y representaciones. En Puente a la vista. Serie de estudios sobre el programa Puente, (No 9). Santiago: FOSIS-Programa Puente-Chile Solidario.

Gradín, Valeria; Picasso, Florencia y Rieiro, Anabel (2012). Participación Ciudadana y Acción Colectiva. Reflexiones sobre políticas institucionales y sujetos colectivos a partir del estudio de tres Casos. En Serna, Miguel et al., Vulnerabilidad y exclusión. Aportes para las políticas sociales, Uruguay Social l Vol. 5, (pp. 35-54). Montevideo: MIDES, Facultad de Ciencias Sociales (FCS)- UdelaR.

Goinheix, Sebastián (2012). La dominación y sus respuestas: Estudio de caso sobre los usos de una política de transferencias condicionadas por parte de sus beneficiarios. Saarbrücken: Académica Española.

Habermas, Jürgen (1992). Teoría de la Acción Comunicativa II. Critica de la razón fundamentalista. Madrid: Taurus.

Habermas, Jürgen (1999). Teoría de la Acción Comunicativa I. Racionalidad de la acción y racionalización social. Madrid: Taurus.

Mik-meyer, Nanna y Villadsen, Kaspar (2014). Power and welfare. Understanding citizens encounters with state welfare. New York: Routledge.

Picasso, Florencia (Coord.) Sosa, Ana; Debellis, Mariela; Goinheix, Sebastián y Cuello, Alexa (2010). Familias y Políticas Sociales en el MERCOSUR y Chile. Rio de Janeiro: Centro Latinoamericano de Economía Humana (CLAEH) Fundación Konrad Adenauer (KAS).

Serna, Miguel et al (2012). Vulnerabilidad y exclusión. Aportes para las políticas sociales. Uruguay Social l Vol. 5, Montevideo: MIDES, UdelaR - FCS.

Weber, Max (1987). Economía y Sociedad. México: Fondo de cultura económica. 